\title{
Avaliação da maturação das fístulas arteriovenosas para hemodiálise pelo eco-Doppler colorido
}

\author{
Evaluation of hemodialysis arteriovenous fistula maturation by \\ color-flow Doppler ultrasound
}

\author{
Jeferson Freitas Toregeani ${ }^{1}$, Claudio Jundi Kimura ${ }^{2}$, Antonio S. Trigo Rocha ${ }^{3}$, Giuliano Giova Volpiani ${ }^{4}$, \\ Ângela Bortoncello ${ }^{5}$, Keity Shirasu ${ }^{5}$, Luiz A. Peres ${ }^{6}$
}

\section{Resumo}

Contexto: Quando se confecciona uma fístula arteriovenosa para hemodiálise $(\mathrm{FAVH})$ autógena, é necessário que se aguarde a dilatação da veia em questão e o desenvolvimento de volume de fluxo mínimo, fenômeno chamado de maturação. Ainda hoje se discute qual o tempo necessário para ocorrer essa maturação.

Objetivo: Avaliar a maturação de FAVH utilizando-se critérios ecográficos.

Método: Entre maio de 2004 e 2005, 40 pacientes foram selecionados prospectivamente, sendo 23 homens $(57,5 \%)$, com média de idade de $17,5 \pm 51,3$ anos, com indicação de confecção de uma FAVH. Utilizou-se o aparelho Logic III ${ }^{\mathbb{R}}$ com transdutor de $10 \mathrm{MHz}$ para a avaliacão no pré-operatório e nos $7^{\circ}, 14^{\circ}, 21^{\circ} \mathrm{e} 28^{\circ}$ dias de pós-operatório. Os critérios para a maturação após a cirurgia foram: veia com diâmetro médio maior que $4 \mathrm{~mm}$ e volume de fluxo maior que $400 \mathrm{~mL} / \mathrm{min}$.

Resultados: O diâmetro médio pré-operatório foi de $3,24 \pm 1,43$ e $3,71 \pm 1,37 \mathrm{~mm}$ para fístulas de punho e de cotovelo, respectivamente. $\mathrm{O}$ diâmetro final foi de $5,01 \pm 0,87 \mathrm{~mm}$ para as FAVH de punho $(\mathrm{p}=0,006)$ e de $6,15 \pm 1,16 \mathrm{~mm}$ para as FAVH de cotovelo $(\mathrm{p}=0,95)$. O volume de fluxo no $7^{\circ}$ dia pós-operatório foi de $493,63 \pm 257,49 \mathrm{~mL} / \mathrm{min}$ e $976,33 \pm 332,90 \mathrm{~mL} / \mathrm{min}$ para as FAVH de punho e cotovelo, respectivamente. Ao final do estudo, foi calculado o valor de $556,81 \pm 288,42 \mathrm{~mL} / \mathrm{min}$ nas FAVH de punho $(\mathrm{p}<0,05)$ e de $1031,62 \pm 614,812 \mathrm{~mL} / \mathrm{min}$ nas FAVH de cotovelo. Baseados nos dois critérios, a maturacão ocorreu em $57,1 \%$ das fístulas de punho e em $100 \%$ das fístulas de cotovelo após a $1^{\mathrm{a}}$ semana. Após 4 semanas, $67,9 \%$ das fístulas de punho e $100 \%$ das fístulas de cotovelo apresentaram maturação.

Conclusão: A maioria das FAVH de cotovelo apresentou diâmetro e fluxo adequados para punção logo após a $1^{\mathrm{a}}$ semana de pós-operatório. Para as FAVH de punho, houve melhora progressiva dos padrões de maturação com o passar das semanas, sugerindo que essas FAVH devem ser puncionadas preferencialmente após a $4^{\mathrm{a}}$ semana de pós-operatório.

Palavras-chave: Fístula, arteriovenosa, hemodiálise, eco-Doppler colorido.

\begin{abstract}
Background: When a hemodialysis arteriovenous fistula (HAVF) is created, it is important to wait for venous dilatation and volume flow increase through the HAVF, a phenomenon called maturation. There is still some controversy as to the exact time required for this maturation to occur.
\end{abstract}

Objective: To evaluate the time required for HAVF maturation using ultrasound criteria.

Method: From May 2004 through May 2005, 40 patients were prospectively selected. The sample was comprised of 23 men $(57.5 \%)$, mean age of $51.3 \pm 17.5$ years, with indication of HAVF creation. Logic III ${ }^{\circledR}$ ultrasound with $10 \mathrm{MHz}$ transducer probe was used for pre- and postoperative evaluation $7,14,21$ and 28 days after the procedure. Criteria for maturation after the procedure were vein diameter larger than $4 \mathrm{~mm}$ and volume flow larger than $400 \mathrm{~mL} / \mathrm{min}$.

Results: Preoperative mean diameter was $3.24 \pm 1.43$ and $3.71 \pm 1.37$ $\mathrm{mm}$ for fist and elbow fistula, respectively. Final diameter of the fist HAVF was $5.01 \pm 0.87 \mathrm{~mm}(\mathrm{p}=0.006)$ and $6.15 \pm 1.16 \mathrm{~mm}$ for the elbow HAVF ( $p=0.95$ ). Flow volume in the 7 th postoperative day was $493.63 \pm 257.49$ and $976.33 \pm 332.90 \mathrm{~mL} / \mathrm{min}$, respectively, for the fist and elbow HAVF. At the end of the study, the value of $556.81 \pm 288.42$ $\mathrm{mL} / \mathrm{min}$ was calculated for the fist HAVF $(\mathrm{p}<0.05)$ and $1,031.62 \pm 614.812 \mathrm{~mL} / \mathrm{min}$ for the elbow HAVF. Based on both cut-off values, maturation occurred in $57.1 \%$ of the fist fistula and in $100 \%$ of the elbow fistula after the first week. After 4 weeks, $67.9 \%$ of the fist HAVF and $100 \%$ of the elbow HAVF presented maturation.

Conclusion: Most elbow HAVF showed adequate diameter and flow volume for puncture 1 week after the procedure. For the fist fistula, gradual maturation occurred throughout the weeks, suggesting that these HAVF should be punctured 4 weeks after the surgery.

Keywords: Fistula, arteriovenous, hemodialysis, color-flow Doppler ultrasound.

1. Cirurgião vascular, Hospital Universitário do Oeste do Paraná, Cascavel, PR. Professor auxiliar, Disciplina de Cirurgia Vascular, Universidade Estadual do Oeste do Paraná (UNIOESTE), Cascavel, PR. Especialista em Cirurgia Vascular, SBACV. Certificado de Atuação em Ecografia Vascular com Doppler, SBACV e Colégio Brasileiro de Radiologia (CBR).

2. Cirurgião vascular, Hospital Universitário do Oeste do Paraná, Cascavel, PR. Certificado de Atuação em Ecografia Vascular com Doppler, SBACV e CBR.

3. Cirurgião vascular, Hospital Universitário do Oeste do Paraná, Cascavel, PR. Professor assistente, Disciplina de Cirurgia Vascular, UNIOESTE, Cascavel, PR. Especialista em Cirurgia Vascular, SBACV. Certificado de Atuação em Ecografia Vascular com Doppler, SBACV e CBR.

4. Cirurgião vascular, Hospital Universitário do Oeste do Paraná, Cascavel, PR.

5. Acadêmico, UNIOESTE, Cascavel, PR. Centro de Estudos, Instituto de Cirurgia Vascular e Angiologia (ICV), Cascavel, PR.

6. Nefrologista, Hospital Universitário do Oeste do Paraná, Cascavel, PR. Professor assistente, Disciplina de Nefrologia, UNIOESTE, Cascavel, PR. Membro da Sociedade Brasileira de Nefrologia.

Não foram declarados conflitos de interesse associados à publicação deste artigo.

Artigo submetido em 10.10.07, aceito em 15.05.08.

J Vasc Bras. 2008;7(3):203-213.

Copyright@ 2008 by Sociedade Brasileira de Angiologia e de Cirurgia Vascular 


\section{Introdução}

As cirurgias de confecção de acessos para hemodiálise são as operações cirúrgicas vasculares mais comuns nos Estados Unidos. A importância de se obter acessos duradouros se deve ao aumento da incidência das doenças renais, da expectativa de vida da população geral e principalmente da população portadora de doença renal crônica $^{1,2}$. Uma boa fístula arteriovenosa para hemodiálise (FAVH) tem que respeitar duas regras básicas para permitir um tratamento adequado ao paciente: ter diâmetro adequado e ter volume de fluxo adequado ${ }^{3}$. Normalmente este diâmetro da veia superficial da FAVH aumenta com o passar das semanas de pós-operatório; o mesmo é observado com relação ao volume de fluxo. Este aumento é variável e depende de fatores como qualidade dos vasos antes da cirurgia, idade do paciente, tributárias, comorbidades, entre outros.

A FAVH autógena, localizada na região do punho, tem sido a primeira escolha para a maioria dos cirurgiões, sendo que a FAVH rádio-cefálica originalmente descrita por Brescia \& Cimino em 1966 ainda hoje é a que confere menor risco de complicações, além de apresentar boa durabilidade ${ }^{4-6}$.

Apesar de ser um procedimento relativamente simples, o planejamento adequado do local da anastomose, os cuidados pré e pós-operatórios e a definição do tempo para início das punções são fundamentais para o sucesso da cirurgia. Os problemas referentes ao planejamento do tratamento do paciente com insuficiência renal já ocorrem em fase pré-dialítica. Alguns autores ${ }^{7}$ indicam a confecção tardia da FAVH, ou seja, após o paciente efetivamente entrar em insuficiência renal terminal (estágio 5), obrigando o paciente a implantar por alguns meses cateteres venosos centrais para hemodiálise ou cateteres de diálise peritoneal até uma FAVH estar pronta para uso. Segundo Bonucchi et al. ${ }^{7}$, o estágio inicial da hemodiálise de preferência é realizado por cateteres venosos centrais, retirando-os assim que a fístula atingir a maturação, que é o nome dado a toda FAVH capaz de ser canulizada e fornecer fluxo mínimo para a máquina de hemodiálise. Em cerca de $40 \%$ desses pacientes, a retirada do cateter venoso central foi possível nos dois primeiros meses tendo em vista a presença de uma FAVH em condições de receber punção.
Outros serviços têm preconizado cada vez mais a indicação precoce da confecção da FAVH, mesmo quando o paciente ainda tem alguma função renal residual (estágios 3 e 4), mas que está em evolução para a fase terminal (estágio 5) ${ }^{7}$. Além disso, a presença de um cateter venoso central como acesso temporário pode interferir na maturação da FAVH, como demonstrado no trabalho de Rayner \& Pisoni ${ }^{8}$. Segundo esses mesmos autores, a confecção da FAVH em estágios mais iniciais da doença diminui a necessidade de punção não programada, que pode levar à falência precoce da fístula $^{8,9}$.

É importante que haja nos serviços de hemodiálise padronização das condutas e criação de serviços de acreditação a fim de se conseguir sucesso na confecção da fístula, onde o ideal são taxas de falência abaixo de $10 \%$. Para tanto, é muito importante o planejamento préoperatório ${ }^{7}$. Vários trabalhos têm demonstrado métodos de se identificar artérias e veias com critérios mínimos adequados para a confecção de uma boa FAVH $^{10,11}$.

Recentemente, o advento do eco-Doppler colorido (EC) facilitou a avaliação pré-operatória dos possíveis vasos doadores e receptores, com possibilidade de identificar, com boa acurácia, a qualidade das veias superficiais (cefálicas e basílicas) e o status do defluxo venoso superficial e profundo, que muitas vezes fica dificultado ao exame clínico. Ao mesmo tempo, o EC permite a análise das características da parede e do fluxo arterial, detectando a presença de alterações, que podem colocar em risco o bom funcionamento da FAVH. Características importantes do método incluem o fato de ter custo relativamente baixo e de não ser invasivo. A associação do EC ao desenvolvimento das FAVH nos serviços de cirurgia vascular tem provocado a melhora dos resultados técnicos deste procedimento ${ }^{1,12}$.

Há controvérsias com relação aos critérios ecográficos para definir uma fístula como madura. Fístulas com mais de $4 \mathrm{~mm}$ e que permitem um fluxo de sangue maior que 250 a $500 \mathrm{~mL} / \mathrm{min}$ podem ser consideradas maduras segundo alguns autores ${ }^{13}$. Por outro lado, ainda é incerto o tempo exato necessário para que ocorra a maturação ${ }^{14}$. Alguns trabalhos demonstraram que a 
Tabela 1 - Critérios de inclusão e exclusão

\begin{tabular}{|c|c|}
\hline Critérios de inclusão & Critérios de exclusão \\
\hline - Insuficiência renal crônica estágios 4 e 5; & $\begin{array}{l}\text { - Necessidade de punção do enxerto antes do término do } \\
\text { estudo; }\end{array}$ \\
\hline - FAV com enxerto nativo; & - Perda de um dos exames; \\
\hline - Quadro clínico estável; & - Não preenchimento de um dos critérios de inclusão. \\
\hline - Diâmetro do lúmen venoso > 2,0 mm; & \\
\hline - Diâmetro do lúmen arterial > 2,0 mm; & \\
\hline $\begin{array}{l}\text { - Ausência de diferencial de pressão arterial > } 20 \text { mmHg entre } \\
\text { os braços; }\end{array}$ & \\
\hline $\begin{array}{l}\text { - Veia superficial continuando para o sistema venoso } \\
\text { profundo; }\end{array}$ & \\
\hline - Ausência de estenoses no sistema venoso central; & \\
\hline $\begin{array}{l}\text { - Ausência de estenoses segmentares ou segmentos arteriais } \\
\text { ocluídos; }\end{array}$ & \\
\hline - Arco de palmar de pérvio (teste de Allen); & \\
\hline $\begin{array}{l}\text { - Disposição para comparecimento a cinco exames de } \\
\text { eco-Doppler colorido; }\end{array}$ & \\
\hline - Autorização para estudo. & \\
\hline
\end{tabular}

$\mathrm{FAV}=$ fístula arteriovenosa

punção precoce (em menos de 2 semanas) deve ser evitada. Entre 2 e 4 semanas, somente em circunstâncias emergenciais deve ser realizada a punção. Existem autores que recomendam que o tempo mínimo necessário para maturação seja de 1 mês e, em algumas circunstâncias, superior a 2 meses $^{4,15,16}$.

A necessidade atual em se concretizar um método barato, de fácil realização e que forneça informações confiáveis sobre o estágio de maturação das FAVH motivou a realização deste trabalho.

\section{Métodos}

No período de 12 meses, entre maio de 2004 e maio de 2005, foram avaliados 40 pacientes, sendo 23 homens e 17 mulheres, com média de idade de 51,3 $\pm 17,5$ anos (variando de 23 a 78 anos), todos com indicação de hemodiálise. Inicialmente foram alocados 76 pacientes, sendo que 36 foram excluídos por falha de preenchimento dos critérios de inclusão ao longo do estudo. Os critérios de inclusão estão representados na Tabela 1.
As fístulas foram confeccionadas no membro nãodominante onde havia a presença de veias pérvias, dando preferência para anastomoses distais no punho (70\%). Todos os pacientes foram submetidos ao exame com EC no pré e no pós-operatório. Ao término do estudo, os pacientes foram liberados para hemodiálise ou para reexploração da fístula em caso de não maturação da mesma.

Os pacientes com hiperuricemia ou hiperpotassemia foram mantidos em hemodiálise por cateter até a maturação da FAVH. Os pacientes que apresentavam valores limítrofes de clearance de creatinina aguardaram para realizar a hemodiálise pela própria FAVH.

Os dados foram expressos como média e desvio padrão e comparados pela análise variância ou teste $\mathrm{F}$ (ANOVA), e foram considerados estatisticamente significativos quando $\mathrm{p}<0,05$.

\section{Técnica do ultra-som}

Utilizou-se o aparelho Logic III ${ }^{\circledR}$ (General Eletric) com transdutor de $10 \mathrm{MHz}$. Todos os pacientes foram examinados na posição sentada, com o braço apoiado 


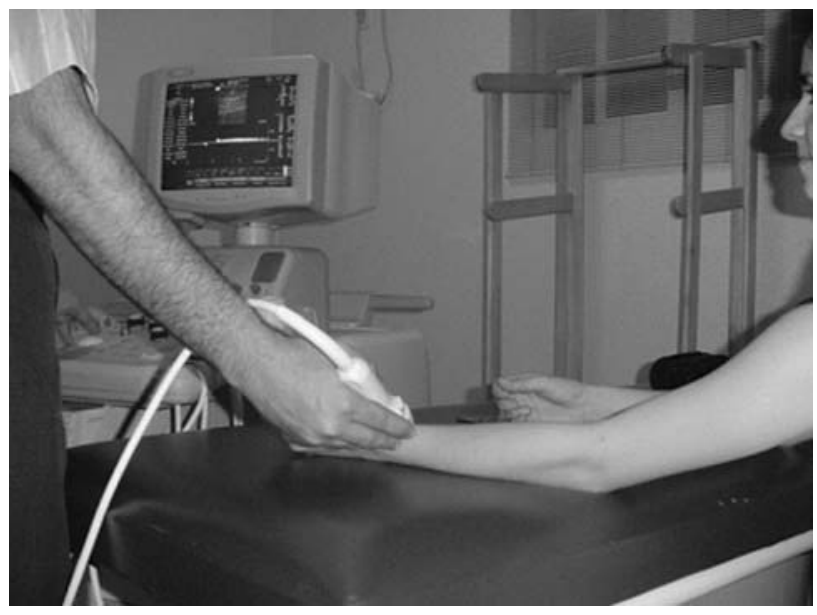

Figura 1 - Posição do paciente para avaliação da fístula arteriovenosa para hemodiálise com o eco-Doppler colorido

sobre a mesa de exame (Figura 1). O ambiente foi mantido à temperatura constante de $25^{\circ} \mathrm{C}$.

A varredura das veias do sistema venoso superficial foi realizada utilizando-se grande quantidade de agente acoplador (gel), com a finalidade de evitar a compressão da veia pelo próprio transdutor. Anotou-se então a compressibilidade e o diâmetro da veia cefálica em vários pontos. Foi avaliada a continuidade do sistema venoso profundo até as veias axilar e subclávia. A seguir, procedeu-se à investigação do diâmetro da artéria radial. Avaliou-se a perviedade do arco palmar através da manobra de Allen. A avaliação do braço dominante só foi realizada quando o não dominante apresentou uma avaliação insatisfatória.

Os pacientes foram reavaliados no $7^{\circ}, 14^{\circ}, 21^{\circ}$ e $28^{\circ}$ pós-operatórios, com mensuração do diâmetro da veia cefálica responsável pelo defluxo da FAVH em pelo menos três pontos. A medição do volume de fluxo foi realizada em um ponto distante o suficiente da anastomose para se evitar o turbilhonamento natural daquela área. Foram realizadas três medidas, posicionando-se o volume-amostra no centro do vaso. A angulação padrão utilizada foi de $60^{\circ}$, corrigida sempre que possível com variações na posição do transdutor. A seguir, o valor do diâmetro do vaso foi medido após o congelamento da imagem, sendo então calculada, automaticamente, a medida do volume de fluxo através de software próprio do aparelho. $\mathrm{O}$ valor final utilizado no estudo foi a média

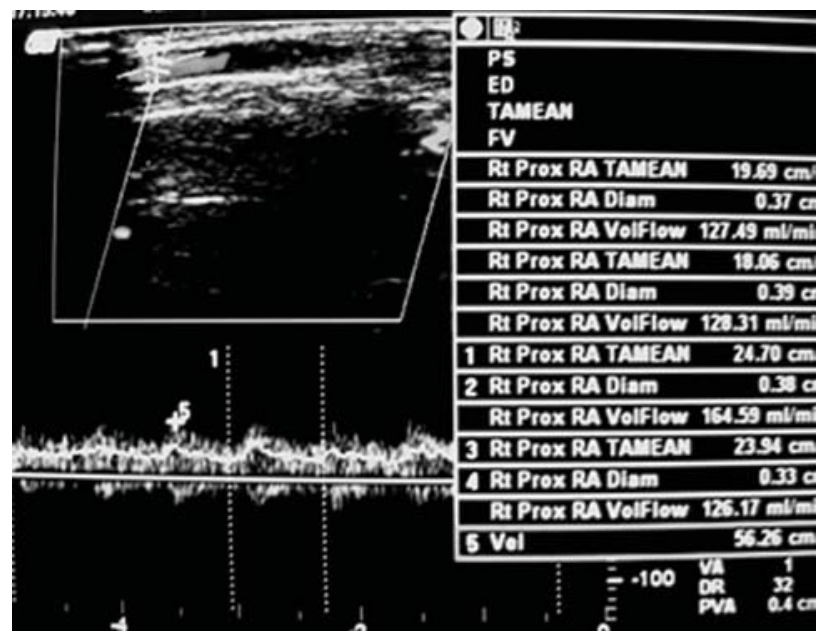

Figura 2 - Foto ilustrativa mostrando aferição do volume de fluxo e diâmetro da fístula arteriovenosa para hemodiálise em no mínimo em três medidas

aritmética de três medidas, tanto de diâmetro quanto de fluxo. Cada exame levou em média 10 minutos (Figura 2). Os critérios ecográficos admitidos para a maturidade da fístula foram veia com diâmetro médio maior que $4 \mathrm{~mm}$ e fluxo maior que $400 \mathrm{~mL} / \mathrm{min}$.

\section{Técnica da cirurgia}

Os pacientes foram submetidos a cirurgia ambulatorial para confecção da FAV utilizando anestesia local com xilocaína $2 \%$, sem vasoconstrictor. Foi realizada incisão longitudinal no punho entre a artéria radial e a veia cefálica, sendo os vasos reparados com alças de silicone. Antes do pinçamento, foi administrada uma solução diluída de heparina (5.000 U diluídas em $250 \mathrm{~mL}$ de soro fisiológico a $0,9 \%$ ) por sonda apropriada, realizando-se a dilatação da veia cefálica por pressão. Procedeu-se ao pinçamento, e foi realizada a arteriotomia longitudinal de cerca de 0,50 a $0,75 \mathrm{~cm}$ e anastomose terminolateral, utilizando-se polipropileno 6-0 ou 7-0. Ao final do procedimento, a veia foi dilatada por massagens externas e por compressão no trajeto proximal. Quando não houve frêmito após a dilatação, foi administrada heparina sistêmica na dose de $3.000 \mathrm{U}$, além de vasodilatadores como a papaverina, conforme descrito por Moreira ${ }^{17}$. A incisão foi fechada com sutura simples. No caso das fístulas de cotovelo, a diferença foi o local da incisão, normalmente oblíqua, na prega do 

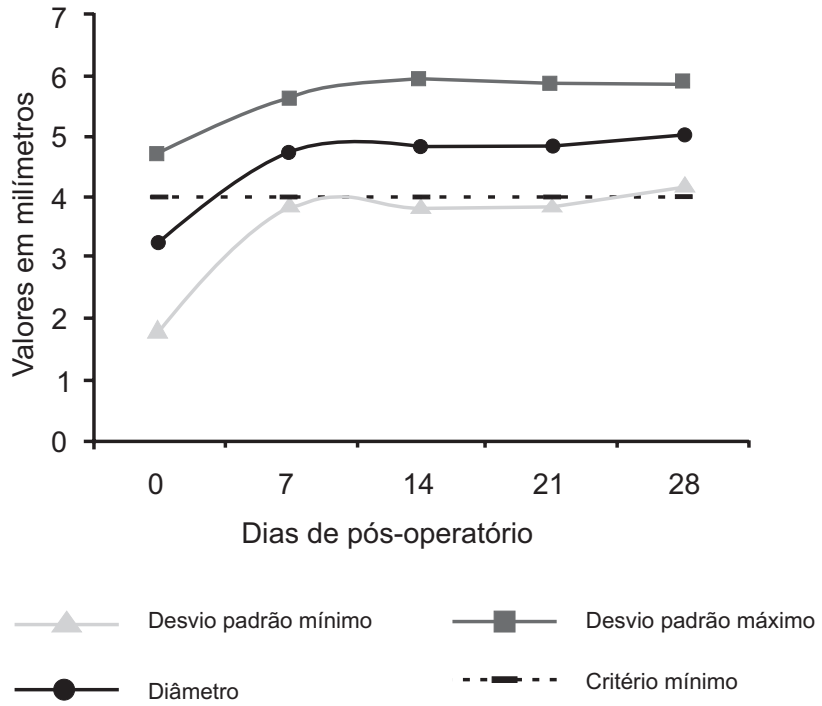

Figura 3 - Variações do diâmetro na fístula arteriovenosa para hemodiálise de punho

cotovelo, onde se dissecou a veia cefálica e a artéria braquial, seguindo o mesmo padrão de anastomose terminolateral com polipropileno 6-0.

\section{Resultados}

O calibre médio pré-operatório foi de $3,24 \pm 1,43$ e $3,71 \pm 1,37 \mathrm{~mm}$ para FAVH de punho e de cotovelo, respectivamente. Houve aumento para 4,73 $\pm 0,87 \mathrm{~mm}$ no $7^{\circ}$ dia pós-operatório nas FAVH de punho $(\mathrm{p}=0,0011)$ e para 5,84 $\pm 1,23 \mathrm{~mm}$ nas FAVH de cotovelo $(\mathrm{p}=0,23)$ no mesmo período, resultando em um aumento percentual do diâmetro de 45,98 e 57,4\%, respectivamente. Esses dados estão representados nas Figuras 3 e 4.

Por volta do $14^{\circ}$ dia pós-operatório, as FAVH de

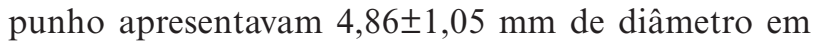
média, e as de cotovelo, $6,02 \pm 1,38 \mathrm{~m}$. Com relação à avaliação pré-operatória, houve significância estatística somente para as FAVH de punho $(\mathrm{p}=0,027)$. No $21^{\circ}$ dia pós-operatório, a medida do diâmetro médio das FAVH de punho foi $4,84 \pm 0,98 \mathrm{~mm}$, e no caso das FAVH de cotovelo, $6,10 \pm 1,30 \mathrm{~mm}$, ambas sem significância estatística ( $p=0,14$ e 0,85 , respectivamente). Ao final do estudo ( $28^{\circ}$ dia pós-operatório), o valor médio para as FAVH de punho foi de $5,01 \pm 0,87 \mathrm{~mm}$, e de cotovelo, $6,15 \pm 1,16 \mathrm{~mm}$. Novamente houve importância, do ponto de vista estatístico, para as variações de diâmetro encontradas nas FAVH de punho $(\mathrm{p}=0,006)$.
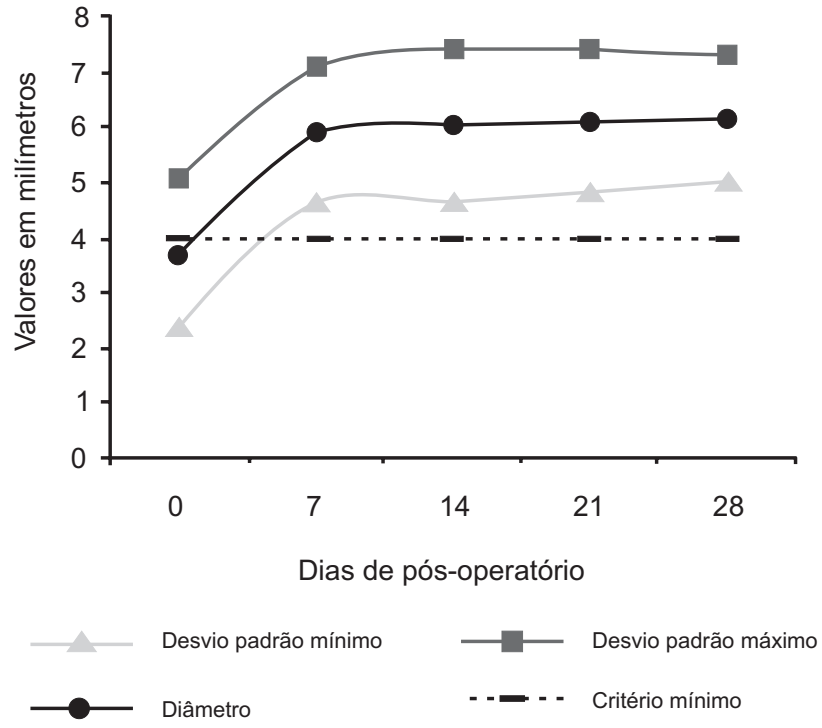

Figura 4 - Variações do diâmetro na fístula arteriovenosa para hemodiálise de cotovelo

A maturação, levando-se em conta o parâmetro de diâmetro maior que 4,0 mm obtido através do EC, ocorreu em $85,7 \%$ das fístulas de punho e em $100 \%$ das fístulas de cotovelo após a $1^{\mathrm{a}}$ semana. No $14^{\circ}$ dia pós-operatório, 78,6\% das FAVH de punho e $91,7 \%$ das FAVH de cotovelo atingiram o critério mínimo, e no $21^{\circ}$ dia após o ato cirúrgico, $75 \%$ das FAVH de punho e 100\% das FAVH de cotovelo apresentavam diâmetros maiores que 4,0 mm. Ao final do estudo, $100 \%$ das fístulas de punho e de cotovelo apresentaram maturação do ponto de vista do diâmetro.

Com relação ao volume de fluxo, os valores préoperatórios foram suprimidos, tendo em vista as baixíssimas velocidades obtidas nas veias superficiais, bem como as grandes variações que as mesmas apresentam ao fluxo, como, por exemplo, as que ocorrem nas fases da respiração. Nesse caso, as comparações foram feitas somente com os dados pós-operatórios, sendo o valor inicial, para análise gráfica, didaticamente considerado como próximo de zero. Com relação às FAVH de punho: no $7^{\circ}$ dia pós-operatório, o volume de fluxo calculado foi de 493,63 $\pm 257,49 \mathrm{~mL} / \mathrm{min}$; no $14^{\circ}$ dia pós-operatório, o volume de fluxo foi de $521,34 \pm 264,44 \mathrm{~mL} / \mathrm{min}$; no $21^{\circ}$ dia pós-operatório, o valor médio na FAVH foi de $458,84 \pm 210,58 \mathrm{~mL} / \mathrm{min}$; e no $28^{\circ} \mathrm{dia}$, foi de $556,81 \pm 288,42 \mathrm{~mL} / \mathrm{min}$. A análise estatística foi significativa somente para as variações ocorridas ao redor da 
Tabela 2 - Percentual de maturação das fístulas arteriovenosas para hemodiálise de punho e de cotovelo com base em critérios obtidos pela medida do diâmetro (critério A), volume de fluxo (critério B) e ambos os critérios (A + B)

\begin{tabular}{cccc}
\hline & \multicolumn{2}{c}{ Percentual de maturação (\%) } \\
\cline { 2 - 4 } & $\begin{array}{c}\text { Diâmetro } \\
\text { (critério A) }\end{array}$ & $\begin{array}{c}\text { Volume de fluxo } \\
\text { (critério B) }\end{array}$ & $\begin{array}{c}\text { Total } \\
\text { (critérios A + B) }\end{array}$ \\
\hline Punho & & & \\
$7^{\circ}$ & 85,7 & 57,1 & 57,1 \\
$14^{\circ}$ & 78,6 & 60,7 & 57,1 \\
$21^{\circ}$ & 75,0 & 57,1 & 57,1 \\
$28^{\circ}$ & 100,0 & 67,9 & 67,9 \\
Cotovelo & & & \\
$7^{\circ}$ & 100,0 & 100,0 & 100,0 \\
$14^{\circ}$ & 91,7 & 91,7 & 91,7 \\
$21^{\circ}$ & 100,0 & 91,7 & 91,7 \\
$28^{\circ}$ & 100,0 & 100,0 & 100,0 \\
\hline
\end{tabular}

$3^{\mathrm{a}}$ semana de pós-operatório $(\mathrm{p}=0,00048)$. Em relação às FAVH de cotovelo: no $7^{\circ}$ dia pós-operatório, o valor calculado para o volume de fluxo foi de $976,33 \pm 332,90$ $\mathrm{mL} / \mathrm{min}$; no $14^{\circ}$ dia pós-operatório, o valor foi de $1137,75 \pm 430,00 \mathrm{~mL} / \mathrm{min} ;$ no $21^{\circ}$ dia pós-operatório, o volume de fluxo médio na FAVH foi de $1151,56 \pm 498,45$ $\mathrm{mL} / \mathrm{min}$; e no $28^{\circ}$ dia, de $1031,62 \pm 614,812 \mathrm{~mL} / \mathrm{min}$. Não houve significância estatística comparando-se os dados calculados nas diversas fases do estudo ( $p>0,05)$.

Do ponto de vista do parâmetro do volume de fluxo mínimo de $400 \mathrm{~mL} / \mathrm{min}, 57,1 \%$ das fístulas de punho e $100 \%$ das fístulas de cotovelo apresentaram maturação após a $1^{\mathrm{a}}$ semana de pós-operatório. No $14^{\mathrm{o}}$ dia pósoperatório $60,7 \%$ das FAVH de punho e $91,7 \%$ das FAVH de cotovelo atingiram o critério mínimo, e no $21^{\circ}$ dia, $57,1 \%$ das FAVH de punho e $91,7 \%$ das FAVH de cotovelo apresentaram volumes de fluxo maiores que 400 $\mathrm{mL} / \mathrm{min}$. Ao final do estudo, $67,9 \%$ das FAVH de punho e 100\% das FAVH de cotovelo apresentaram maturação do ponto de vista do volume de fluxo.

A Tabela 2 mostra as porcentagens de FAVH maturadas quando analisados o diâmetro e o volume de fluxo em conjunto.

Quatro pacientes com FAVH de punho que não atingiram os parâmetros mínimos de maturação foram submetidos à nova exploração da FAVH. Depois de identificado o escape de fluxo através de algumas tributárias calibrosas, foi realizada a ligadura dessas tributárias, com melhora dos parâmetros de maturação no pós-operatório.

Quanto a problemas pós-operatórios, não observamos nenhuma infecção ou qualquer outra complicação cirúrgica.

\section{Discussão}

As FAVH confeccionadas com material autógeno têm sido as mais comumente utilizadas, pois apresentam perviedade primária e secundária melhor do que a FAVH confeccionada com enxerto sintético ${ }^{18,19}$. Enxertos ou cateteres apresentam menor sobrevida devido às complicações isquêmicas, maior incidência de trombose e oclusão por hiperplasia intimal, além de maior susceptibilidade a infecções, oferecendo maior morbimortalidade para o paciente quando comparada com uma FAVH autógena ${ }^{20}$. As FAVH nativas constituem o melhor acesso vascular para hemodiálise, segundo o National Kidney Foundation ${ }^{21}$.

Alguns serviços têm preconizado o uso mais freqüente de fístulas proximais (cotovelo), tendo em vista as melhores taxas de perviedade em longo prazo em relação às FAVH de punho ${ }^{7}$. No entanto, as FAVH distais costumam gerar menos alterações hemodinâmicas para 
o sistema circulatório, trazendo, portanto, mais segurança para o paciente.

Devido ao fato de apresentarem diâmetros menores, os vasos distais (artéria radial e veia cefálica, principalmente) necessitam de uma avaliação prévia à cirurgia, para que somente vasos com um mínimo de qualidade sejam utilizados, evitando assim cirurgias desnecessárias, que geralmente resultam em fracasso técnico. $\mathrm{Na}$ literatura, é difícil encontrar um trabalho consistente que demonstre o valor mínimo que uma veia deve ter de diâmetro a fim de ser utilizada na confecção da FAVH. Alguns autores postulam um valor mínimo de $1,8 \mathrm{~mm}$; outros, $2,0 \mathrm{~mm}^{4,10,22}$. Com relação à artéria doadora ocorre o mesmo, sendo que o diâmetro mínimo de 2,0 $\mathrm{mm}$ tem sido utilizado. Segundo Malovrh ${ }^{16}$, há um risco aumentado de falência precoce da FAVH quando o diâmetro interno da artéria doadora é menor que 1,6 $\mathrm{mm}$. Pode-se ainda acrescentar o pico sistólico mínimo de 50 $\mathrm{cm} / \mathrm{s}$ como valor de corte, e o cálculo do índice de resistência (RI) da artéria radial antes e após um teste de hiperemia reativa, utilizando-se a ultra-sonografia Doppler colorida. A presença de um RI > 0,7 na artéria radial indica uma artéria com baixa probabilidade de sofrer dilatação ${ }^{4}$.

Vários grupos documentaram o valor do uso de métodos de imagem não-invasivos antes e após a construção dos acessos vasculares. Segundo o Dialysis Outcomes Quality Initiative (DOQI), a avaliação através do EC é importante, pois além de o exame ser não-invasivo, permite a fácil avaliação das veias superficiais, proporciona a análise espectral do fluxo arterial e permite o acompanhamento do desenvolvimento do diâmetro e do volume de fluxo da FAVH (maturação), melhorando o resultado técnico das mesmas ${ }^{19,23}$. FAVH avaliadas pelo EC apresentam menor incidência de falha precoce e melhor perviedade em longo prazo ${ }^{1}$. O uso da ultrasonografia Doppler colorida pode identificar algum tipo de anormalidade em aproximadamente $40 \%$ dos $\operatorname{casos}^{23}$. Surratt et al. ${ }^{24}$ enfatizaram a importância de exames mais aprofundados naqueles casos suspeitos, como a venografia pré-operatória. Em um estudo recente realizado por esses autores, $40 \%$ de todos os pacientes com histórico de cateter venoso central apresentavam estenoses significativas na origem das veias subclávia e/ou jugular.
A maturação das fístulas arteriovenosas usualmente ocorre num período variável de 4 a 12 semanas. Neste período ocorrem vários fenômenos, como o aumento da artéria aferente e o aumento do fluxo sangüíneo arterial. Do lado venoso também ocorre a dilatação, a arterialização e o aumento global de fluxo pela FAVH. A arterialização venosa consiste no espessamento parietal venoso ocasionado pela proliferação das células musculares lisas da parede vascular, tornando a veia menos susceptível à compressão extrínseca. Essa série de modificações é responsável pela chamada maturação da fístula, permitindo as punções repetidas e o uso de curativos compressivos após a retirada da agulha coletora, sem, no entanto, obstruir a veia ${ }^{25}$. Embora muito se tenha publicado sobre a maturação, a quantidade de análises que permitem conclusões mais acertadas é pequena $^{7,21}$.

Para entender o que é maturação, é necessário conhecer a hemodinâmica, a anatomia, a estrutura molecular e funcional dos vasos envolvidos. Uma das alterações hemodinâmicas mais importantes é o aumento das forças de cisalhamento que ocorrem tanto na artéria aferente como na veia responsável pelo defluxo da $\mathrm{FAVH}^{21}$.

Para calcular a força de cisalhamento $(\tau)$, utiliza-se a fórmula matemática $\tau=6 \mu \mathrm{Q} / \mathrm{wh}^{2}$, onde $\mu=$ viscosidade sangüínea, $\mathrm{Q}=$ fluxo do sangue, $\mathrm{w}=$ largura $\mathrm{e} \mathrm{h}=$ altura $^{26}$.

Vasos com fluxo laminar elevado apresentam forças de cisalhamento elevadas. Vasos com fluxo lento, multidirecional, têm forças de cisalhamento baixas. Assim, o corpo humano sempre tenta manter a força de cisalhamento dentro do normal. Se ocorrer um aumento da velocidade do sangue e, conseqüentemente, da força de cisalhamento, o vaso responde com dilatação, já que a viscosidade não se altera, acarretando em diminuição das forças em questão ${ }^{21}$.

Em um estudo de Corpataux et al. ${ }^{3}$, obteve-se, através de técnicas ultra-sonográficas, a aferição do volume de fluxo, do diâmetro e espessura da parede vascular, bem como o cálculo das forças de cisalhamento. $\mathrm{O}$ valor médio de $539 \mathrm{~mL} / \mathrm{min}$ foi encontrado na $1^{\text {a }}$ semana após a confecção da FAVH (325 a $990 \mathrm{~mL} / \mathrm{min}$ ), resultando em um aumento da força de cisalhamento para $24,5 \mathrm{dyn} / \mathrm{cm}^{2}$ - em uma veia normal do antebraço, este 
parâmetro não passa de 5 a $10 \mathrm{dyn} / \mathrm{cm}^{2}$, ou seja, um aumento de quase três vezes. Tal resultado foi acompanhado por um aumento no diâmetro interno do vaso da veia cefálica de 2,37 para 4,43 mm em 1 semana de funcionamento. Ao final da $4^{\mathrm{a}}$ semana de estudo, o diâmetro da veia aumentou para $5,04 \mathrm{~mm}$ e, após o $3^{\circ}$ mês, chegou a um diâmetro máximo de $6,62 \mathrm{~mm}$. Como as forças de cisalhamento são inversamente proporcionais ao diâmetro do vaso, ocorre uma diminuição gradual para níveis próximo do normal $\left(18,1 \mathrm{dyn} / \mathrm{cm}^{2}\right.$ na $4^{\mathrm{a}}$ semana e $10,4 \mathrm{dyn} / \mathrm{cm}^{2}$ na $12^{\mathrm{a}}$ semana). Um aumento progressivo foi observado na secção transversal da veia cefálica, aumentando de $4,4 \mathrm{~mm}^{2}$ em 1 semana para 5,3 $\mathrm{mm}^{2}$ em 4 semanas e $6,9 \mathrm{~mm}^{2}$ em 12 semanas $(\mathrm{p}=0,028)$, indicando um aumento da massa vascular. Neste trabalho, os resultados foram semelhantes, indicando que as principais alterações de volume e fluxo ocorrem já na $1^{\text {a }}$ semana de pós-operatório.

Em nível biológico, a dilatação vascular ocorre devido ao aumento das forças de cisalhamento, que acabam por causar alinhamento das células endoteliais e estímulo à liberação de fatores de relaxamento, como o óxido nítrico e prostaciclinas, que também promovem inibição da agregação plaquetária ${ }^{3}$. Em contraste, forças de cisalhamento baixas acabam por ativar o endotélio, estimulando a liberação de fatores pró-trombóticos e vasoconstrictores, como as tromboxanas ${ }^{3}$.

Existe também relação com a função das metaloproteases que digerem componentes da matriz extracelular e de fatores de crescimento como o platelet derived growth factor (PDGF) neste processo. Alguns autores demonstraram que a retirada do endotélio vascular causa a supressão dessas respostas ${ }^{3}$.

A tentativa do vaso de reduzir as forças de cisalhamento às vezes é barrada pela hiperplasia medial que ocorre na veia da FAVH, levando à redução da luz e a um conseqüente aumento da força de cisalhamento. Portanto, após a confecção da FAVH, estas duas forças interagem, resultando nas variações das forças de cisalhamento, que levarão ou não à maturação da FAVH $^{3}$.

Cerca de 30\% das fístulas nunca apresentarão maturação, seja por baixo fluxo na artéria aferente, seja por falta de dilatação na veia de defluxo. Esses valores podem melhorar caso haja um sistema de avaliação préoperatória no qual se consiga detectar vasos de pequeno calibre ou com estenoses naturais ${ }^{6}$. A taxa de falência para FAVH primária pode ser reduzida significativamente, como visto por Silva et al. ${ }^{1}$, que observaram redução da taxa de falência de $36 \%$ para $8 \%$ após a implementação de um protocolo para avaliação préoperatória, fato que aumentou o uso de FAVH primárias para $64 \%$. Segundo Tordoir et al. ${ }^{27}, 10$ a $24 \%$ das cirurgias para confecção das FAVH falham, seja por trombose ou por falha na maturação.

A falha na maturação na FAVH pode ocorrer devido à ausência de dilatação da artéria aferente. Isso é bem evidente em indivíduos diabéticos e/ou urêmicos onde a calcificação exagerada impede a secreção de mediadores que promovam a vasodilatação. A falha pode ocorrer também em função de problemas na porção venosa da FAVH, que, devido a punções prévias ou processos inflamatórios, ou mesmo alterações constitucionais, tornam a veia fibrótica e anulam sua capacidade de dilatação. Por fim, a incapacidade de atingir a maturação pode vir a acontecer semanas ou meses após a confecção da FAVH, devido a alterações nas forças de cisalhamento no local da anastomose, resultando em pontos onde essas forças são muito baixas, levando à hiperplasia intimal e obstrução da FAVH ${ }^{3}$. Segundo Brunori et al. ${ }^{28}$, a maturação não deve seguir um critério isolado, como sugerido por alguns autores, que estabeleceram o prazo de 14 dias como data limite. A preferência de Brunori et al. ainda é de analisar cada caso individualmente e deixar a fístula maturar por pelo menos 30 dias. Outros autores sugerem um mínimo de 1 mês e, preferencialmente, 2 a 3 meses antes de se canulizar uma FAVH recém confeccionada ${ }^{19}$.

Alguns serviços realizam a avaliação pós-operatória das fístulas por exame físico simples, procurando canulizar a FAVH após 4 a 6 semanas. Nesses casos, são consideradas maduras as fístulas nas quais o exame mostrou veias facilmente visíveis e/ou palpáveis e canulização fácil. As FAVH que se apresentaram de forma diferente são encaminhadas para avaliação por métodos invasivos. O sucesso clínico, além da canulização fácil, deve constar de volume de fluxo mínimo de $500 \mathrm{~mL} / \mathrm{min}^{6}$. 
Em uma análise recente, Saran et al. ${ }^{29}$ analisaram questionários preenchidos por enfermeiras que trabalhavam em unidades de hemodiálise. De acordo com os dados, em hospitais japoneses, 74\% das FAVH haviam sido canulizadas antes do $30^{\circ}$ dia pós-operatório; já em hospitais europeus, o resultado foi de $50 \%$; finalmente, em hospitais americanos, somente $2 \%$ das FAVH foram canulizadas antes do $30^{\circ}$ dia.

Segundo Basile et al. ${ }^{30}$, fístulas maduras, mas com volume de fluxo menor que $700 \mathrm{~mL} / \mathrm{min}$, devem ser acompanhadas de perto, haja vista a incidência significativamente maior de falência em médio prazo.

Alterações do volume de fluxo na FAVH ocorrem logo nos primeiros minutos após a anastomose. Vários autores demonstraram essas alterações através da análise com ultra-sonografia Doppler. Em um estudo de 50 FAVH, Won et al. ${ }^{31}$ identificaram um aumento do volume de fluxo de 20,9 $\mathrm{mL} / \mathrm{min}$ para $174 \mathrm{~mL} / \mathrm{min} 10$ minutos após a confecção da FAVH. Em outro estudo, Yerdel et al. ${ }^{32}$ mostraram que as variações continuam ocorrendo ao longo das semanas, com aumento do volume de fluxo para 754,799 e $946 \mathrm{~mL} / \mathrm{min}$ no $1^{\circ}, 7^{\circ} \mathrm{e}$ $42^{\circ}$ dia de pós-operatório.

Robin et al. ${ }^{33}$ publicaram dados referentes às alterações de volume de fluxo e diâmetro que ocorrem nos vasos após a confecção de uma FAVH. As principais ocorrem cedo, logo nas primeiras semanas. Após 4 semanas, um diâmetro médio de 4,5 mm e um volume de fluxo de $700 \mathrm{~mL} / \mathrm{min}$ foram encontrados. Após 12 semanas, os valores modificaram-se pouco, para $4,7 \mathrm{~mm}$ de diâmetro e $675 \mathrm{~mL} / \mathrm{min}$ no volume de fluxo. O estudo de Robin et al. ${ }^{33}$ determinou um fator importante: o diâmetro venoso mínimo de $4,0 \mathrm{~mm}$ e um volume de fluxo mínimo de $500 \mathrm{~mL} / \mathrm{min}$ foram associados a fístulas maturadas em 70\% dos casos. Associando-se as duas variáveis, quando o paciente apresentava estes dois requisitos mínimos, 95\% das fístulas eram adequadas para uso. Esses valores permaneceram com pouca variação após o $2^{\circ}, 3^{\circ}$ e $4^{\circ}$ meses de pós-operatório.

Vários estudos demonstraram que boa parte das fístulas que não atingem um dos parâmetros mínimos de diâmetro $(4,0 \mathrm{~mm})$ e volume de fluxo $(500 \mathrm{~mL} / \mathrm{min})$ acabam por fechar em um prazo de 6 a 8 semanas $^{27,33,34}$.
Outros autores acreditam que volumes de fluxo menores podem permitir hemodiálise, dependendo do ajuste da máquina de hemodiálise. Um exemplo foi o trabalho de Tordoir et al. ${ }^{27}$, onde um volume de fluxo de 250 $\mathrm{mL} / \mathrm{min}$ foi considerado adequado para maturação. Para alguns autores, o volume de fluxo mínimo na FAVH deve ser de $300 \mathrm{~mL} / \mathrm{min}$, evitando-se desta forma colabamento durante a hemodiálise ${ }^{4}$. No trabalho de Sivanesan et al. ${ }^{35}$, um diâmetro mínimo de $3 \mathrm{~mm}$ e um volume de fluxo mínimo de $150 \mathrm{~mL} / \mathrm{min}$ foi considerado suficiente para maturação. Em nossa casuística, o valor de corte foi definido em $400 \mathrm{~mL} / \mathrm{min}$, com base no fato de que o fluxo mínimo para que a máquina de hemodiálise trabalhe razoavelmente é de $350 \mathrm{~mL} / \mathrm{min}$. FAVH com valores iguais ou abaixo de $400 \mathrm{~mL} / \mathrm{min}$ geralmente evoluem com colabamento da veia e interrupção do processo.

Pela análise da literatura, demonstrou-se que as alterações de diâmetro e volume de fluxo ocorrem precocemente, entre 1 e 4 semanas. Assim, acredita-se que em 6 semanas a fístula deve ser avaliada para punção, pois nesse período, as FAVH que apresentam problemas de maturação já podem ser identificadas ${ }^{3,36}$.

Neste estudo, a maturação ecográfica ocorreu em mais de $50 \%$ dos pacientes já na $1^{\mathrm{a}}$ semana. No entanto, sabe-se que este é um critério insuficiente, pois muitos dos pacientes que necessitaram de punção precoce da FAVH acabaram perdendo-a. Neste exemplo, temos dois pacientes que infelizmente tiveram perda da FAVH por uma quebra do protocolo. Ambos desenvolveram hiperpotassemia associada à infecção do cateter temporário, necessitando retirada do cateter e tentativa frustrada de punção precoce da FAVH, resultando em hematoma e oclusão da FAVH, mesmo que estas apresentassem os critérios mínimos presentes.

Nos casos em que a FAVH não teve desenvolvimento adequado devido a fatores passíveis de correção, o eco-Doppler colorido mostrou-se eficaz na identificação precoce desses fatores. Como exemplo, tivemos um caso de escape por tributárias, com fluxo sangüíneo médio de $250 \mathrm{~mL} / \mathrm{min}$. Após a ligadura das tributárias, houve melhora do padrão do fluxo da FAVH, que chegou a $824 \mathrm{~mL} / \mathrm{min}$. 
Uma equipe treinada e sem muitos recursos adicionais pode determinar se uma fístula atingiu a maturação ou não. Robins et al. ${ }^{33}$ publicaram um trabalho em que se comparou a avaliação clínica da maturação da FAVH por critérios como frêmito facilmente palpável e trajeto de pelo menos $10 \mathrm{~cm}$ de veia passível de punção. Os dados foram cruzados com dados obtidos através de avaliação ultra-sonográfica da maturação das FAVH. Trinta pacientes foram examinados. Das 21 FAVH clinicamente maduras, 17 (81\%) realmente apresentavam-se maturadas ecograficamente. Por outro lado, sete de nove FAVH (78\%) foram avaliadas pelas enfermeiras como inadequadas, e realmente o eram. Assim, a acurácia de um examinador experiente gira em torno de $80 \%$.

Em um estudo publicado por Tordoir et al. ${ }^{27}$ a falha na maturação ocorreu em 33\% das FAVH, sendo que em $64 \%$ das fístulas que não maturaram foi identificada uma causa anatômica passível de correção por técnicas recentes de tratamento endovascular. Essas técnicas, quando utilizadas, permitiram o salvamento de $47 \%$ das FAVH com problemas de maturação devido a alterações anatômicas localizadas.

O exame físico pode determinar as causas de falência na maturação. Um pulso arterial fraco pode pressupor uma estenose acima do nível da FAVH. O escape através de tributárias é uma situação freqüente, impedindo um volume de fluxo adequado pela veia principal. Uma forma de identificá-la é comprimindo a veia que causa o suposto escape de fluxo e percebendo as variações de frêmito (exame físico) ou as variações de volume de fluxo (com ultra-som), que indicam se o escape é significativo ou não e se aquele vaso pode ser ligado $^{3}$.

Um fato percebido em nossa casuística é que se deve evitar a ligadura de tributárias que supostamente drenam parte do sangue proveniente da FAVH antes de se realizar o teste de compressão da tributária. Através desse teste, é possível determinar se o escape é significativo ou não, devendo-se indicar a ligadura nos casos positivos. Nos casos cujo escape não é significativo, deve-se manter a tributária, pois quando ocorre a oclusão da fístula, esses vasos podem manter a veia cefálica pérvia, permitindo a confecção de novos acessos para hemodiálise com trajetos remanescentes.

\section{Conclusão}

Pelos critérios ecográficos definidos para o valor de corte entre FAVH maturadas ou não, conclui-se que a maioria das FAVH de cotovelo apresentou diâmetro e fluxo adequados para punção logo após a $1^{\mathrm{a}}$ semana de pós-operatório. Para as FAVH de punho, houve melhora progressiva dos padrões de maturação com o passar das semanas, sendo que estas devem ser puncionadas de preferência após a $4^{\mathrm{a}}$ semana de pós-operatório.

\section{Agradecimentos}

Nossos agradecimentos à equipe de nefrologia da Renalclin, de Cascavel, Dra. Hi Yung, Dra. Noris Rhode, Dr. Mauricio Carvalho e Dra. Vanessa Uoscocowich, e também à enfermeira Solange Santos e à funcionária Claudinete Balbino Alvarez.

\section{Referências}

1. Silva MB Jr., Hobson RW 2nd, Pappas PJ, et al. A strategy for increasing use of autogenous hemodialysis access procedures: Impact of preoperative noninvasive evaluation. J Vasc Surg. 1998;27:302-7; discussion 307-8.

2. Vanholder R. Vascular access: care and monitoring of function. Nephrol Dial Transplant. 2001;16:1542-5.

3. Corpataux JM, Haesler E, Silacci P, Ris HB, Hayoz D. Lowpressure environment and remodelling of the forearm vein in Brescia-Cimino haemodialysis access. Nephrol Dial Transplant. 2002;17:1057-62.

4. Wiese P, Nonnast-Daniel B. Colour Doppler ultrasound in dialysis access. Nephrol Dial Transplant. 2004;19:1956-63.

5. Jennings WC. Creating arteriovenous fistulas in 132 consecutive patients: exploiting the proximal radial artery arteriovenous fistula: reliable, safe, and simple forearm and upper arm hemodialysis access. Arch Surg. 2006;141:27-32.

6. Clark WI, Cohen RA, Kwak A, et al. Salvage of nonmaturing native fistulas by using angioplasty. Radiology. 2007;242(1):286-92.

7. Bonucchi D, D’Amelio A, Grosoli M, Baraldi A, Capelli G. Vascular access for haemodialysis: from surgical procedure to an integrated therapeutic approach. Nephrol Dial Transplant. 1998;13 Suppl 7:78-81.

8. Rayner RL, Pisoni RL, Gillespie BW, et al. Creation, cannulation and survival of arteriovenous fistulae: data from the Dialysis Outcomes and Practice Patterns Study. Kidney Int. 2003;63:323-30.

9. Pisoni RL, Young EW, Dykstra DM, et al. Vascular access use in Europe and the United States: results from the DOPPS. Kidney Int. 2002;61:305-16. 
10. Planken RN, Keuter XH, Hoeks AP, et al. Diameter measurements of the forearm cephalic vein prior to vascular access creation in end-stage renal disease patients: graduated pressure cuff versus tourniquet vessel dilatation. Nephrol Dial Transplant. 2006;21:802-6.

11. Allon M, Lockhart ME, Lilly RZ, et al. Effect of preoperative sonographic mapping on vascular access outcomes in hemodialysis patients. Kidney Int. 2001;60:2013-20.

12. Grogan J, Castilla M, Lozanski L, Griffin A, Loth F, Bassiouny $\mathrm{H}$. Frequency of critical stenosis in primary arteriovenous fistulae before hemodialysis access: Should duplex ultrasound surveillance be the standard of care? J Vasc Surg. 2005;41(6):1000-6.

13. Huber TS, Ozaki CK, Flynn TC, et al. Prospective validation of an algorithm to maximize native arteriovenous fistulae for chronic hemodialysis access. J Vasc Surg 2002; 36: 452-9.

14. Berman SS, Gentile AT. Impact of secondary procedures in autogenous arteriovenous fistula maturation and maintenance. J Vasc Surg. 2001;34:866-71.

15. Saran R, Pisoni RL, Young EW. Timing of first cannulation of arteriovenous fistula: are we waiting too long? Nephrol Dial Transplant. 2005;20:688-90.

16. Malovrh M. Native arteriovenous fístula: preoperative evaluation. Am J Kidney Dis. 2002;39:1218-25.

17. Moreira RCR. Fístulas arteriovenosas para diálise: algumas lições aprendidas ao longo de 1.200 operações. Rev Angiol Cirur Vasc. 1997;6:206-11.

18. Mendes RR, Farber MA, Marston WA, Dinwiddie LC, Keagy BA, Burnham SJ. Prediction of wrist arteriovenous fistula maturation with preoperative vein mapping with ultrasonography. J Vasc Surg. 2002;36:460-3.

19. III. NKF-K/DOQI Clinical Practice Guidelines for Vascular Access: update 2000. Am J Kidney Dis. 2001;37(1 Suppl 1):S137-81.

20. Duval L. Proximal radial artery arteriovenous fistulae (PRAAVF). Nephrol Nurs J. 2007;34:217-8.

21. Asif A, Roy-Chaudhury P, Beathard GA. Early arteriovenous fistula failure: a logical proposal for when and how to intervene. Clin J Am Soc Nephrol. 2006;1:332-9.

22. Patel ST, Hughes J, Mills JL Sr.. Failure of arteriovenous fistula maturation: an unintended consequence of exceeding dialysis outcome quality initiative guidelines for hemodialysis access. J Vasc Surg. 2003;38:439-45.

23. Huber TS, Ozaki CK, Flynn TC, et al. Prospective validation of an algorithm to maximize native arteriovenous fistulae for chronic hemodialysis access. J Vasc Surg. 2002;36:452-9.

24. Surratt RS, Picus D, Hicks ME, Darcy MD, Kleinhoffer M, Jendrisak M. The importance of preoperative evaluation of the subclavian vein in dialysis access planning. AJR Am J Roentgenol. 1991;156:623-5.
25. Haimovici H, Ascer E, Hollier LH, Strandness DE Jr., Towne JB. Haimovici's vascular surgery. 4th ed. UK: Blackwel Science. 1996. p. 913-37.

26. Chiu JJ, Chen LJ, Lee PL, et al. Shear stress inhibits adhesion molecule expression in vascular endothelial cells induced by coculture with smooth muscle cells. Blood. 2003;101:2667-74.

27. Tordoir JH, Rooyens P, Dammers R, van der Sande FM, de Haan M, Yo TI. Prospective evaluation of failure modes in autogenous radiocephalic wrist access for haemodialysis. Nephrol Dial Transplant. 2003;18:378-83.

28. Brunori G, Ravani P, Mandolfo S, Imbasciati E, Malberti F, Cancarini G. Fistula maturation: doesn't time matter at all? Nephrol Dial Transplant. 2005;20:684-7.

29. Saran R, Dykstra DM, Pisoni RL, et al. Timing of first cannulation and vascular access failure in haemodialysis: an analysis of practice patterns at dialysis facilities in the DOPPS. Nephrol Dial Transplant. 2004;19:2334-40.

30. Basile C, Ruggieri G, Vernaglione L, Montanaro A, Giordano $\mathrm{R}$. The natural history of autogenous radio-cephalic wrist arteriovenous stulas of haemodialysis patients: a prospective observational study. Nephrol Dial Transplant. 2004;19:1231-6.

31. Won T, Jang JW, Lee S, Han JJ, Park YS, Ahn JH. Effects of intraoperative blood flow on the early patency of radiocephalic fistulas. Ann Vasc Surg. 2000;14:468-72.

32. Yerdel MA, Kesenci M, Yazicioglu KM, Doseyen Z, Turkcapar AG, Anadol E. Effect of haemodynamic variables on surgically created arteriovenous fistula flow. Nephrol Dial Transplant. 1997;12:1684-8.

33. Robbin ML, Chamberlain NE, Lockhart ME, et al. Hemodialysis arteriovenous fistula maturity: US evaluation. Radiology. 2002;225:59-64.

34. Lin SL, Huang CH, Chen HS, Hsu WA, Yen CJ, Yen TS. Effects of age and diabetes on blood flow rate and primary outcome of newly created hemodialysis arteriovenous fistulas. Am J Nephrol. 1998;18:96-100.

35. Sivanesan S, How TV, Bakran A. Characterizing flow distributions in AV fistulae for haemodialysis access. Nephrol Dial Transplant. 1998;13:3108-10.

36. Beathard GA, Arnold P, Jackson J, Litchfield T; Physician Operators Forum of RMS Lifeline. Aggressive treatment of early fistula failure. Kidney Int. 2003;64:1487-94.

Correspondência:

Jeferson Freitas Toregeani

Rua Dom Pedro II, 2359

CEP 85812-120 - Cascavel, PR

Tel.: (45) 3225.1288

E-mail: jeferson@vascularpr.com.br 